\title{
COMMUNICATION
}

\section{Graphene Oxide Membranes with Tunable Permeability due to Embedded Carbon Dots}

Cite this: DOI: $10.1039 / \times 0 \times x 00000 x$

\author{
Wentai Wang, ${ }^{a, c}$ Ehsan Eftekhari, ${ }^{a}$ Guangshan Zhu, ${ }^{a}$ Xiwang Zhang, ${ }^{b^{*}}$ Zifeng Yan, ${ }^{{ }^{*}}$ \\ and Qin $\mathrm{Li}^{a^{*}}$
}

Received 00th January 2012,

Accepted 00th January 2012

DOI: $10.1039 / \times 0 \times x 00000 x$

www.rsc.org/

In this paper, we fabricated graphene oxide membranes with tunable permeation by embedding carbon nanodots of controllable sizes.

Membranes are useful tools for the separation of liquids or gases. ${ }^{1,2}$ Among various materials that can be applied to fabricate membranes, the majority are polymers (e.g. cellulose, polyamide, polymethyl methacrylate, etc.). ${ }^{2}$ However polymeric membranes generally have poor chemical and thermal stability, which limits their application. Graphene and its derivative graphene oxide (GO) are an emerging two-dimensional material with single atom layer, ${ }^{3}$ which have attracted significant attention as membrane materials. ${ }^{4}$ Graphene/GO membranes exhibit desirable properties for membranes including high solute rejection rate, excellent mechanical strength, good flexibility, chemical and thermal stability, ${ }^{5}$ which makes them highly interesting and promising for water treatment and gas separation..$^{6-10}$

It has been reported that the paper-like graphene/GO membranes can be assembled via solvent-casting or vacuum filtration of colloidal dispersions of graphene or GO. ${ }^{11,12}$ The channels in graphene/GO membranes are the interspace between individual nanosheets, hence their membrane pore sizes are in theory between 0.4-1.3 nm, ${ }^{9}$ falling in the category of reverse osmosis or nanofiltration. Therefore, graphene/GO are considered as effective filtration membranes in water purification and predicted to offer much higher throughputs than state-of-the-art membranes in water desalination. $^{13}$ In addition, GO membranes were found to have advantageous properties in antibacterial and antifouling functions, ${ }^{14-}$ ${ }^{16}$ selective transport of alkali and alkaline earth cations ${ }^{17}$ and efficient separation of macromolecules and particles, ${ }^{12}$ which are highly desirable in water purification. However, due to the substrate/drying-induced flattening effect, ${ }^{12}$ graphene or GO debris stack layer by layer in a flattened form leading to a very tightly stacked structure without much room between the layers. The lack of channels in GO membranes leads to low water permeation, hence poor water flux rate, which greatly limits their practical applications. $^{18}$

To improve the permeability of $\mathrm{GO} /$ graphene membranes, various approaches have been developed to create more space and channels between $\mathrm{GO} /$ graphene layers, for example, insertion of small molecules or nanostrands into the layers, 5,19 or morphology modification by creating controlled corrugations in reduced GO (rGO) layers through either chemical treatment ${ }^{12}$ or drying process. ${ }^{20}$ Although these methods can successfully achieve the modulation of graphene/GO membranes interlayer space, there are still limitations such as untunable pore size, much reduced mechanical strength, complex procedure, and high cost.

Herein, we fabricated GO membranes with carbon dots (CDs) embedded into the interspaces to tune the spacing and channels between GO layers and evaluated its performance on filtration. CDs are recently discovered quasi-spherical, fluorescent nanocarbons that comprise amorphous or graphitic or polymeric carbon nanoparticles, typically with abundant hydrophilic groups such as hydroxyl and carboxyl groups on surface. ${ }^{21-24}$ In addition, the size of CDs can be precisely controlled in the range of $1-5 \mathrm{~nm}$ in an inexpensive and facile manner. ${ }^{21}$ This work, to our best knowledge, is the first study on tuning the pore sizes of GO membranes by embedding CDs which have similar properties as GO. The compositional similarity between $\mathrm{GO}$ and $\mathrm{CDs}$ results in a better distribution of CDs on GO sheets compared to other type of nanoparticles. The fabrication process of CDs embedded GO (CDs-GO) membranes is illustrated in Scheme 1.

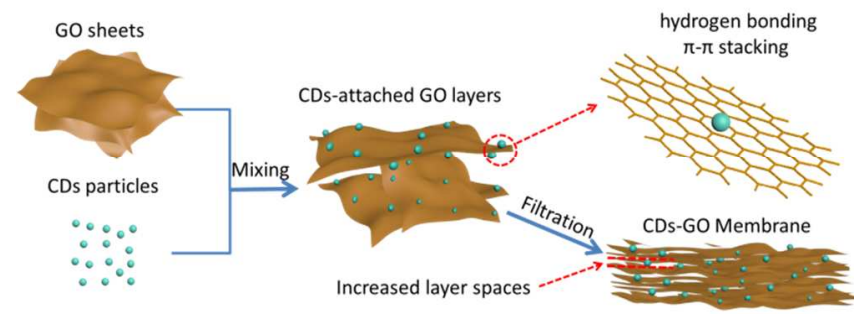

Scheme 1 The fabrication process of CDs-GO membranes.

Nitrogen-doped CDs were synthesized and fractionated ${ }^{25}$ into three sizes, namely $<1 \mathrm{kDa}, 1 \sim 3.5 \mathrm{kDa}$ and $>3.5 \mathrm{kDa}$, defined by the molecular weight cut-off (MWCO) size of the dialysis membranes. The atomic force microscopy (AFM) scans show that their sizes are centred at 1, 5 and $8 \mathrm{~nm}$, respectively, as displayed in Fig. 1A-C, which are confirmed by the overlaid dynamic light scattering (DLS) measurement results. The AFM image in Fig. 1D shows the morphology of the as-synthesized GO nanosheets, which are of a thickness of about $1 \mathrm{~nm}$, consistent with earlier reports. ${ }^{26}$ Upon vacuum filtration, the mixture of $\mathrm{CDs}$ and GO nanosheets self- 
assembled into uniformly layered structure as depicted in Scheme 1. scanning electron microscope (SEM) images of CDs-GO membrane reveal the slightly wrinkled GO membrane surface (Fig. 1E) and the clearly layered feature in cross-section (Fig.1F). The digital photograph of a typical CDs-GO membrane of $5 \mathrm{~cm}$ in diameter is displayed in the inset of Fig. 1E. The dimples on the membrane are due to the vacuum suction force through the holes on the Buchner funnel.

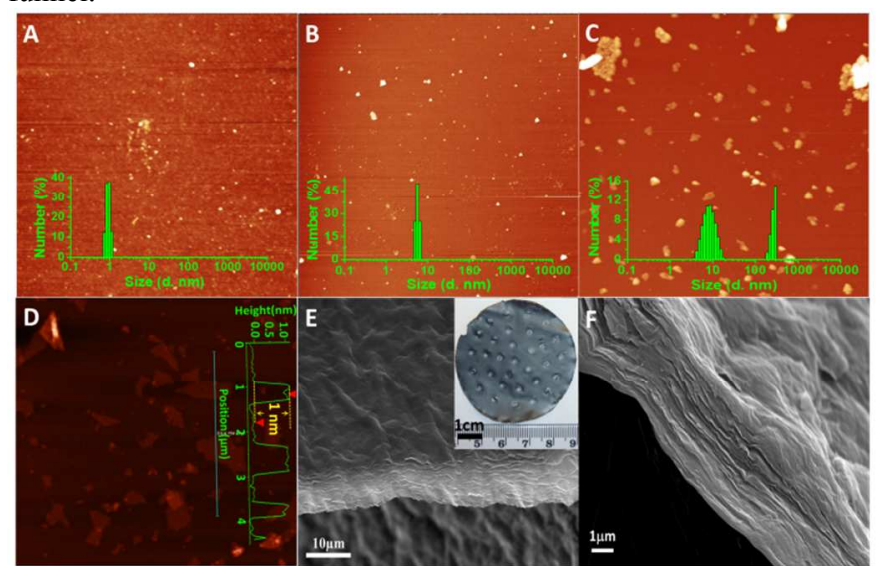

Fig.1 A-C) AFM images of the three fraction of CDs with MWCO $<1 \mathrm{k}$, $1 \sim 3.5 \mathrm{k}$ and $>3.5 \mathrm{k}$ Da; Overlay: particle size determined by DLS; D) AFM image of GO with height profile along line; E) The SEM image of CDs-GO membrane; inset: optical photograph of CDs-GO membrane (30 ml GO, 15

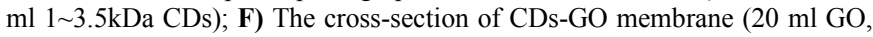
$15 \mathrm{ml}$ CDs with MWCO 1 3.5 k Da).

The thickness of CDs-GO membranes using different loading of GO were measured by SEM as shown in Fig. S1, confirming that the membrane thickness can be controlled by adjusting the loading of GO suspension. Typically, $15 \mathrm{ml}$ (containing about $36 \mathrm{mg}$ of GO) of GO suspension was used and the ratio of GO to CDs was adjusted by the volume of CDs suspension. The thickness of CDs-GO membranes that we used in the following measurements and application tests was about $2.5 \mu \mathrm{m}$.

There were no noticeable differences in Fourier transform infrared spectroscopy (FTIR) and Raman spectra between GO and CDs-GO membranes as shown in Fig. S2. This can be attributed to the similarity of $\mathrm{CDs}$ and $\mathrm{GO}$ in chemical composition. Both are comprised primarily of carbon with abundant hydroxyl and carboxyl groups. X-ray photoelectron spectroscopy (XPS) surface scan shows a presence of nitrogen in CDs-GO and no nitrogen in GO membrane (Fig. S2). The total nitrogen percentage in the CDs-GO membrane is about $0.18 \%$, which is an evidence of a successful incorporation of CDs in the GO membranes. AFM scan of a CDs-GO membrane surface also shows the presence of CDs on GO surface (Fig. S5).

The specific surface area and pore size of GO and CDs-GO membranes cannot be acquired through the nitrogen adsorptiondesorption technique, possibly due to the disappearance of interlayer space as a result of the degassing process, which prevents the permeation and adsorption of $\mathrm{N}_{2} .{ }^{9}, 27$ However, since the GO membranes are permeable to water, ${ }^{27}$ the mass loss of the wet membranes after drying was employed to determine the membrane porosity as shown in Table 1 . The porosities of the CDs-GO membranes are $42-171 \%$ higher than those of their counterpart GO membranes. The increased porosity further evidences that the CDs have been successfully embedded between GO nanosheets and significantly increased the interspaces between the nanosheets.

It is known that an electrical double layer is formed around GO nanosheets in water due to the oxygen containing groups, which is a key factor in the interaction between GO and other nanoparticales. ${ }^{28}$ On the other hand, CDs have very similar chemical structure as GO- like materials. ${ }^{21,29}$ Therefore, upon mixing CDs and GO suspensions together, CDs tend to attach onto the surface of GO sheets possibly through hydrogen bonding. During drying, the attachment of CDs on GO sheets may be enhanced by van der Waals force and $\pi-\pi$ stacking. Moreover, the presence of GO layer surfaces appears to be able to prevent the agglomeration of CDs, leading to a uniform distribution of CDs on GO layers. These CDs-attached GO layers self-assembled piece by piece in an ordered form by vacuum filtration. ${ }^{30}$

The water flux through the CDs-GO and GO membranes were assessed. The results show significantly improved permeability in CDs-GO membranes as displayed in Fig. 2A. Under a transmembrane pressure of $0.1 \mathrm{MPa}$, the water flux through GO membrane was only $53 \mathrm{~L} / \mathrm{h} \cdot \mathrm{m}^{2}$, but reached as high as $439 \mathrm{~L} / \mathrm{h} \cdot \mathrm{m}^{2}$ after embedding CDs with MWCO $>3.5 \mathrm{kDa}$. The improved flux is much higher than that the thermal expanded GO paper ${ }^{18}$ whose highest permeation is only about $205 \mathrm{~L} / \mathrm{h} \cdot \mathrm{m}^{2}$ at $0.1 \mathrm{MPa}$. With increase in the size of CDs, the water flux increased accordingly. This direct correlation confirms that it is the embedded CDs that increased the distance between GO layers, forming enlarged nanochannels.

\begin{tabular}{ccccc}
\multicolumn{4}{c}{ Table 1 Porosity of GO membranes embedded with different sizes of CDs. } \\
\hline $\begin{array}{c}\text { Membranes } \\
\text { Type }\end{array}$ & GO & $\begin{array}{c}\text { CDs-GO } \\
\text { (<1 kDa) }\end{array}$ & $\begin{array}{c}\text { CDs-GO } \\
(\mathbf{1} \sim \mathbf{3 . 5} \text { kDa) }\end{array}$ & $\begin{array}{c}\text { CDs-GO } \\
(>\mathbf{3 . 5} \text { kDa) }\end{array}$ \\
\hline $\begin{array}{c}\text { Void Ratio } \\
\text { (\%) }\end{array}$ & 9.5 & 13.5 & 23.3 & 25.7 \\
\hline
\end{tabular}
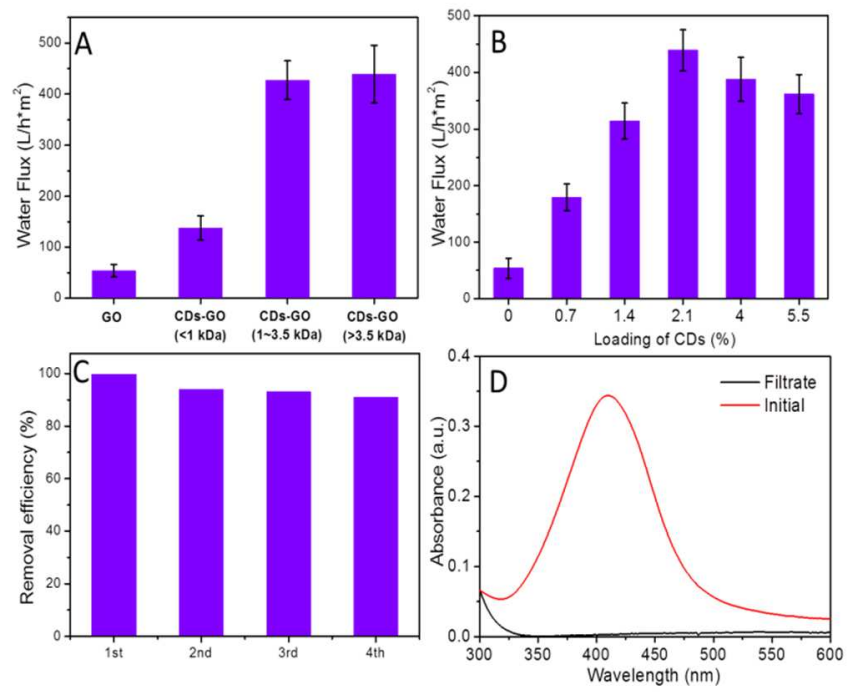

Fig.2 A) The permeability of GO and CDs-GO membranes embedded with CDs of different size (Note: CDs amount is about $2.1 \%$ ); B) The loading of CDs on the permeability of CDs-GO membranes (CDs: $1 \sim 3.5 \mathrm{kDa}$ ); C) The cycle tests of CDs-GO membranes on MO removal; D) The spectra of $\mathrm{Ag}$ NPs before and after filtration.

We further investigated the effect of the loading of CDs $(1 \sim 3.5$ $\mathrm{kDa}$ ) on the permeability of CDs-GO membranes. As shown in Fig. $2 \mathrm{~B}$, the water permeability increased with the increase in loading of CDs initially. The optimal loading of CDs in terms of permeability was about $2.1 \%$ (weight of CDs/total weight of CDs-GO). Further increasing $\mathrm{CDs}$ loading resulted in a decline in membrane permeability, which suggests that when the CDs are over-loaded, the CDs may occupy the interspace and reduce the available nanochannels, leading to the decrease of water permeability of CDs-GO membranes.

The potential application of the CDs-GO membranes for water filtration was tested. The results enlisted in Table 2 show the 
effective removal ability of GO and CDs-GO membranes for small molecules (MB, MO and $\mathrm{RhB}$ ). The dye removal efficiency can reach over $99 \%$ by GO, CDs-GO $(<1 \mathrm{kDa})$ and CDs-GO $(1 \sim 3.5 \mathrm{kDa})$ membranes, which indicate that the increased water permeability of the CDs-GO membranes $(<1 \mathrm{kDa}$ and $1 \sim 3.5 \mathrm{kDa})$ does not compromise their performances in small molecule removal. A 3 5\% decrease in dye removal efficiency of CDs-GO (>3.5 kDa) membranes was observed, which may be because that some channels formed by the $>3.5 \mathrm{kDa}$ CDs are larger than the target pollutants. According to the results, the CDs-GO membranes made of CDs of $1 \sim 3.5 \mathrm{kDa}$ is the most promising as it possesses both high permeability and high dye removal percentage.

The cycling tests of the CDs-GO membranes on dye removal were also performed using $\mathrm{MO}$ as the model molecule to assess the durability of the membranes (Fig. 2C). The used CDs-GO membrane was washed with ethanol to remove the residual $\mathrm{MO}$ on membranes before each subsequent test. The removal efficiency of MO slightly decreased from $99.8 \%$ to $90.4 \%$ after 4 cycles, which may be due to the poor stability of GO membrane in water; as GO is hydrophilic, the interspace between GO nanosheets can increase over time, leading to reduced rejection.

To evaluate the separation property of CDs-GO membranes for nanoparticles, Ag nanoparticles (NPs) of $6 \mathrm{~nm}$ (DLS data shown in Fig. S3) were prepared ${ }^{31}$ and filtrated through CDs-GO membranes. The rejection rate of $\mathrm{Ag}$ NPs was determined to be $99.4 \%$ through the absorbance measured by UV-Vis as demonstrated in Fig. 2D. It demonstrates that NPs with size larger than $6 \mathrm{~nm}$ can be efficiently rejected by the CDs-GO membranes.

Table 2 The parameters of dye (MB, MO and RhB) solution filtration by GO and CDs-GO membranes.

\begin{tabular}{|c|c|c|c|c|}
\hline Membrane & Dye & $\begin{array}{l}\text { Molecular } \\
\text { Size }(\AA)\end{array}$ & $\begin{array}{c}\text { Permeability } \\
\left(\mathrm{L} / \mathrm{h} \cdot \mathrm{m}^{2}\right)\end{array}$ & $\begin{array}{c}\text { Removal } \\
\text { Efficiency } \\
(\%)\end{array}$ \\
\hline \multirow{3}{*}{ GO } & MB & 6 & 51 & 99.9 \\
\hline & $\mathrm{MO}$ & 8 & 53 & 99.9 \\
\hline & RhB & 8 & 46 & 99.9 \\
\hline \multirow{3}{*}{$\begin{array}{l}\text { CDs-GO } \\
<1 \mathrm{kDa}\end{array}$} & MB & 6 & 137 & 99.9 \\
\hline & $\mathrm{MO}$ & 8 & 123 & 99.9 \\
\hline & RhB & 8 & 119 & 99.9 \\
\hline \multirow{3}{*}{$\begin{array}{c}\text { CDs-GO } \\
1 \sim 3.5 \mathrm{kDa}\end{array}$} & MB & 6 & 434 & 99.5 \\
\hline & $\mathrm{MO}$ & 8 & 408 & 99.2 \\
\hline & RhB & 8 & 422 & 99.7 \\
\hline \multirow{3}{*}{$\begin{array}{c}\text { CDs-GO } \\
>3.5 \text { kDa }\end{array}$} & MB & 6 & 421 & 94.1 \\
\hline & MO & 8 & 447 & 96.6 \\
\hline & RhB & 8 & 439 & 96.9 \\
\hline
\end{tabular}

Owing to the chemical similarity between $\mathrm{CDs}$ and GO, we can readily extend this technique to fabricate $\mathrm{rGO}$ membranes, which are more stable in water, with tunable permeability. Here we demonstrate the preparation of CDs-rGO (CDs size 1 3.5 kDa) membrane by performing $\mathrm{HI}$ reduction ${ }^{32}$ on its parent CDs-GO membrane. CDs-rGO membrane appeared fluffier than rGO membrane (Fig. S4). The presence of embedded CDs has increased the permeability of rGO membrane from $18 \mathrm{~L} / \mathrm{h} \cdot \mathrm{m}^{2}$ to $204 \mathrm{~L} / \mathrm{h} \cdot \mathrm{m}^{2}$. The more than 10 fold increase in permeability in CDs-rGO suggests the incorporation of CDs was also suitable for graphene membranes.

\section{Conclusions}

In summary, we have demonstrated a facile approach to fabricate GO membranes with tunable permeability by embedding CDs. The layer space between GO can be controlled by the size of CDs, and therefore, tunes the permeability of GO membranes. The similar carbon structures between GO and
CDs allow a more compatible integration of the two materials, forming stable membranes. We have also demonstrated that the CDs-GO membranes are highly efficient in removing organic pollutants and rejection of nanoparticles (e.g. larger than $6 \mathrm{~nm}$ ). Moreover, the method presented here can be readily extended to graphene membranes. The study provides new insight to fabrication of nanostructured membranes and application for water treatment.

\section{Acknowledgement}

X. Zhang thanks Australia Research Council (DP110103533) and Monash University for his ARF and Larkins fellowships.

\section{Notes and references}

${ }^{a}$ Queensland Micro- and Nanotechnology Centre, Griffith University Nathan Campus, Brisbane, Australia. Fax: 617555 28226; Tel: 617 3735 7514; E-mail: qin.li@griffith.edu.au

${ }^{b}$ Department of Chemical Engineering, Monash University Clayton Campus, Melbourne, Australia. E-mail: xiwang.zhang@monash.edu

${ }^{C}$ State Key Laboratory of Heavy Oil Processing Key Laboratory of CNPC, China University of Petroleum, Qingdao, China. E-mail: zfyancat@upc.edu.cn

$\dagger$ Electronic Supplementary Information (ESI) available: experimental details, SEM images on thickness, FTIR, Raman and XPS spectra, porosity data. See DOI: 10.1039/c000000x/

1. H. Strathmann, AIChE Journal, 2001, 47, 1077.

2. A. K. Pabby, S. S. H. Rizvi, A. M. Sastre, Handbook of Membrane Separations: Chemical, Pharmaceutical, Food, and Biotechnological Applications, 2008, 3, 25.

3. X. Li, X. Wang, L. Zhang, S. Lee, H. Dai, Science, 2008, 319, 1229.

4. D. Li and R. B. Kaner, Science, 2008, 320, 1170.

5. H. Huang, Z. Song, N. Wei, L. Shi, Y. Mao, Y. Ying, L. Sun, Z. Xu, X. Peng, Nature Commun., 2013, 4, 2979.

6. H. Li, Z. Song, X. Zhang, Y. Huang, S. Li, Y. Mao, H. J. Ploehn, Y. Bao and M. Yu, Science, 2013, 342, 95.

7. H. W. Kim, H. W. Yoon, S. M. Yoon, B. M. Yoo, B. K. Ahn, Y. H. Cho, H. J. Shin, H. Yang, U. Paik, S. Kwon, J. Y. Choi and H. B. Park, Science, 2013, 342, 91.

8. B. Mi, Science, 2014, 343, 740.

9. R. K. Joshi, P. Carbone, F. C. Wang, V. G. Kravets, Y. Su, I. V. Grigorieva, H. A. Wu, A. K. Geim, R. R. Nair, Science, 2014, 343, 752.

10. Y. Han, Z. Xu and C. Gao, Adv. Funct. Mater., 2013, 23, 3693.

11. D. A. Dikin, S. Stankovich, E. J. Zimney, R. D. Piner, G. H. Dommett, G. Evmenenko, S. T. Nguyen and R. S. Ruoff, Nature, 2007, 448, 457.

12. L. Qiu, X. Zhang, W. Yang, Y. Wang, G. P. Simon and D. Li, Chem. Commun., 2011, 47, 5810.

13. E. N. Wang and R. Karnik, Nature Nanotechnol., 2012, 7, 552.

14. C. Cheng, S. Li, S. Nie, W. Zhao, H. Yang, S. Sun, C. Zhao, Biomacromolecules, 2012, 13, 4236.

15. C. Cheng, S. Nie, S. Li, H. Peng, H. Yang, L. Ma, S. Sun, C. Zhao, J. Mater. Chem. B, 2013, 1, 265.

16. W. Hu, C. Peng, W. Luo, M. Lv, X. Li, D. Li, Q. Huang, and C.Fan, ACS Nano, 2010, 4, 4317.

17. P. Sun, F. Zheng, M. Zhu, Z. Song, K.Wang, M. Zhong, D. Wu, R. B. Little, Z. Xu, and H. Zhu, ACS Nano, 2014, 8, 850. 
18. J. Zhu, L. Zhu, Z. Lu, L. Gu, S. Cao and X. Cao, J. Phys. Chem. C, 2012, 116, 23075.

19. S. Stankovich, D. A. Dikin, O. C. Compton, G. H. B. Dommett, R. S. Ruoff and S. T. Nguyen, Chem. Mater., 2010, 22, 4153.

20. W. Lv, Z. Li, G. Zhou, J.J. Shao, D. Kong, X. Zheng, B. Li, F. Li, F. Kang, Q. H. Yang, Adv. Funct. Mater., 2014, 24, 3456.

21. S. N. Baker and G. A. Baker, Angew. Chem., 2010, 49, 6726.

22. Y. P. Sun, B. Zhou, Y. Lin, W. Wang, K. A. S. Fernando, P. Pathak, M. J. Meziani, B. A. Harruff, X. Wang, H. Wang, P. G. Luo, H. Yang, M. Erkan Kose, B. Chen, L. M. Veca, S. Y. Xie, J. Am. Chem. Soc., 2006, 128, 7756.

23. P. Zhang, W. Li, X. Zhai, C. Liu, L. Dai, W. Liu, Chem. Commun., 2012, 48, 10431.

24. R. Liu, D. Wu, S. Liu, K. Koynov, W. Knoll, Q. Li, Angew. Chem., 2009, 48, 4598.

25. C. Wang, X. Wu, X. Li, W. Wang, L. Wang, M. Gu, Q. Li, J.Mater. Chem., 2012, 22, 15522.

26. Z. Luo, Y. Lu, L. A. Somers, A. T. C. Johnson, J. Am. Chem. Soc., 2009, 131, 898.

27. R. R. Nair, H. A. Wu, P. N. Jayaram, I. V. Grigorieva, A. K. Geim, Science, 2012, 335, 442.

28. F. Bei, X. Hou, S. L. Chang, G. P. Simon, D. Li, Chemistry, 2011, 17, 5958.

29. Y. Dong, J. Shao, C. Chen, H. Li, R. Wang, Y. Chi, X. Lin, G. Chen, Carbon, 2012, 50, 4738.

30. K. W. Putz, O. C. Compton, C. Segar, Z. An, S. T. Nguyen, and L. C. Brinson, ACS Nano, 2011, 5, 6601.

31. J. P. Ge, W. Chen, L. P. Liu, Y. D. Li, Chemistry, 2006, 12, 6552.

32. S. Pei, J. Zhao, J. Du, W. Ren, H.-M. Cheng, Carbon, 2010, 48, 4466. 